[13] E. B. Meigs, N. R. Bhatherwice et C. A. Cary. Jour. Biol. Chem., 1919, $37,1$.

[14] Ch. Porcher, Rapport au Congrès national de Laiterie à Rennes, oct. 1922, édité par l'Industrie laitière.

\title{
CARENCE MINÉRALE CHEZ LES VACHES LAITIERES(1)
}

\section{par}

\section{le Professeur RAdu VLADESCO}

Laboratoire de Chimie biologique de la Faculté de Médecine vétérinaire de Bucarest, Roumanie.

A l'occasion de quelques recherches relatives au métabolisme du phosphore dans l'organisme animal, notre attention fut attirée par une constatation assez intéressante: la présence des phosphates d'une manière constante et dans des proportions assez élevées dans les urines de vaches appartenant à une institution publique et abritées dans une même étable.

On sait que, dans les conditions normales, l'urine des herbivores contient seulement des traces de phosphates et que des quantités plus notables se rencontrent dans les états fiévreux, dans les catarrhes de l'intestin, dans l'inanition, et surtout dans l'ostéomalacie et le rachitisme.

Or, l'examen attentif de l'état général des animaux ne nous permit pas de découvrir des signes apparents d'une maladie quelconque.

D'autre part, en faisant une enquête sur le rendement en lait de quelques individus, nous apprîmes qu'il était très précaire.

En effet, pendant un laps de temps dépassant deux mois et assez éloigné du commeneement et de la fin de la période de lactation, le rendement moyen journalier chez une vache, sur laquelle notre attention fut fixée spécialement, fut de 2 litres 29 pendant un mois, arec un maximum de 2 litres 8 et un minimum de 1 litre; et de 1 litre 93 pendant le mois suivant, avec un maximum de 2 litres 4 et un minimum de 1 litre.

Ce dernier fait nous fit penser à une ration alimentaire insuffisante ou mal équilibrée.

Les investigations faites dans cette direction nous montrèrent que l'hypothèse formulée ci-dessus était juste.

En effet, les fourrages mis à la disposition des animaux appartenant à l'étable mentionnée étaient les suivants : paille de millet, orge broyé, son de froment.

Chaque animal recevait en moyenne : $11 \mathrm{~kg}$. 66 de paille, $2 \mathrm{~kg}$. 66 d'orge, $1 \mathrm{~kg}$. 66 de son.

(1) Paru dans le Volume jubilaire du Professeur Ch. Pcrcher. 
Supposons pour l'instant que la eomposition chimique des aliments utilisés est la même que celle donnée dans les tableaux de KELLNER.

Dans ces tahleaux, on trouve :

\begin{tabular}{|c|c|c|c|c|c|c|c|c|c|c|c|c|}
\hline in) 78 f & 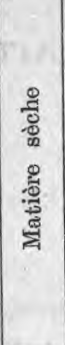 & 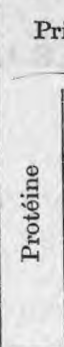 & 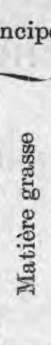 & 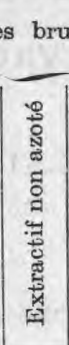 & $\frac{8}{0}$ & 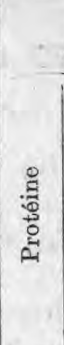 & 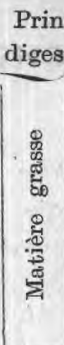 & $\frac{\text { cipes }}{\text { tibles }}$ & $\frac{8}{\frac{8}{3}}$ & 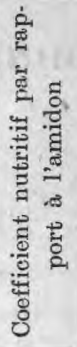 & 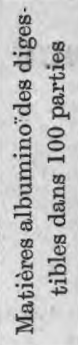 & 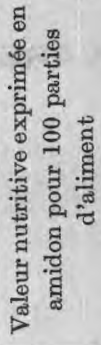 \\
\hline Paille ..... & 85,0 & 4,8 & 2,3 & 36,4 & 35,2 & 1,6 & 1,1 & 20 & 19,4 & 0,52 & 1,2 & 22,3 \\
\hline $\begin{array}{l}\text { Graines d'or- } \\
\text { ge (moyen.) }\end{array}$ & 85,7 & 9,4 & 2,1 & 67,8 & 3,9 & 6,6 & 1,9 & 62,4 & 1,3 & 0,99 & 6,1 & 72,0 \\
\hline $\begin{array}{r}\text { Son de fro- } \\
\text { ment } \ldots .\end{array}$ & 87,8 & 14,3 & 4,2 & 52,2 & 10,2 & 11,3 & 3 & 37,1 & 2,6 & 0,77 & 9,8 & 42,6 \\
\hline
\end{tabular}

En ne tenant compte que de trois des desiderata réclamés pai l'alimentation rationnelle des animaux, à savoir :

$1^{0}$ la proportion de matière sèche,

$2^{\circ}$ la valeur nutritive en amidon,

$3^{\circ}$ la relation nutritive en amidon,

on peut, à l'aide des trois équations suivantes, déterminer les quan. tités de chacun de ces aliments, dont la somme puisse constituer une ration suffisante.

Soit $x, y, z$, les quantités de paille, orge, et son.

On a :

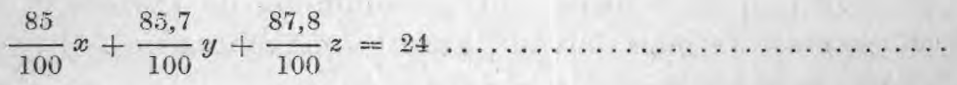

(C'est la quantité de matière sèche admise pour les vaches laitières donnant par jour 5 litres de lait et pour $1.000 \mathrm{~kg}$. de poids vif.)

$$
\frac{22,3}{100} x+\frac{72}{100} y+\frac{42,6}{100} z=8,5
$$

(C'est la valeur en amidon.)

$$
\frac{\frac{1,6}{100} x+\frac{6,6}{100} y+\frac{11,3}{100} z}{2,4\left(\frac{1,1}{100} x+\frac{1,9}{100} y+\frac{3}{100} z\right)+\frac{39,4}{100} x+\frac{63,7}{100} y+\frac{39,7}{100} z}=\frac{1}{8,5} \ldots
$$


(C'est la relation nutritive.) -

Les valeurs trouvées sont :

$$
\begin{aligned}
& x \text { (paille) }=17,33 \\
& y \text { (orge) }=0,447 \\
& z \text { (son) }=10,13
\end{aligned}
$$

Pour une vache de $500 \mathrm{~kg}$. : $8 \mathrm{~kg}$. 660 de paille, $0 \mathrm{~kg}$. 224 d'orge, $5 \mathrm{~kg} .065$ de son.

En examinant de près les chiffres qui représentent les quantités de fourrages offertes en réalité : $11 \mathrm{~kg}$. 66 de paille, $2 \mathrm{~kg}$. 66 d'orge, $1 \mathrm{~kg}$. 66 de son, on trouve que la ration était suffisante en ce qui concerne la valeur nutritive (exprimée en amidon).

Elle était par contre insuffisante en ce qui concerne le besoin en azote.

On pourrait objecter certainement que la composition chimique des fourrages utilisés ne peut pas être identique à celle trouvée dans les tableaux de KeLLiN BiR.

L'objection est justifiée et elle est d'ailleurs confirmée par les résultats des analyses concernant les substances protéiques.

En effet, nous avons trouvé les teneurs suivantes pour les protéines brutes :

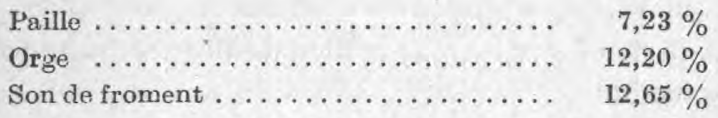

En prenant comme coefficients de digestibilité : $0,33,0,70,0,80$ (valeurs établies expérimentalement sur les ruminants pour les protéines de ces trois fourrages), on trouve un total de 0 gr. 675 de protéine digestible.

Or, cette quantité est suffisante pour couvrir les nécessités de l'organisme en azote. (Voir les tableaux de rationnement.)

Quoi qu'il en soit, la troisième condition envisagée (relation nutritive) n'est pas remplie.

La ration telle qu'on l'a instituée (11 kg. 66 de paille, 2 kg. 66 d'orge, $1 \mathrm{~kg}$. 66 de son), a une relation nutritive de $\frac{1}{13,5}$, et avec la correction dont il est question ci-dessus, elle s'élève seulement jusqu'à $\frac{1}{11,1}$.

La relation nutritive reste assez éloignée de celle qui est admise, c'est-à-dire de $\frac{1}{8,5}$.

La relation alimentaire était done mal équilibrée.

Peut-on attribuer le fait signalé au commencement de cette note 
- la phosphaturie des vaches daitières (nous avons trouvé des quantités de $\mathrm{P}^{2} \mathrm{O}^{5}$ s'élevant jusqu'à 3 gr. par litre d'urine) - à ce déséquilibre alimentaire?

Nous croyons qu'on ne peut pas l'affirmer d'une manière certaine, sans d'autres recherches.

Pour enlever le doute qui pourrait subsister, nous avons poussé plus loin nos investigations dans cette direction, en déterminant les teneurs en chaux et en acide phosphorique des fourrages qui entraient dans la composition de la ration alimentaire (1).

Voici les résultats de ces analyses :

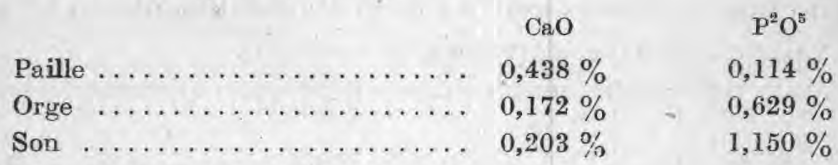

Avec ces chiffres, on trouve dans la ration administrée : $57 \mathrm{gr} .30$ de chaux et 51 gr. 10 d'acide phosphorique.

En comparant ces taux à ceux considérés actuellement comme nécessaires pour une vache laitière qui donne cinq litres de lait par jour et ayant un poids vif de $500 \mathrm{~kg}$. : $100 \mathrm{gr}$. de chaux et $75 \mathrm{gr}$. d'acide phosphorique $\left(=54,30 \quad \mathrm{P}^{2} \mathrm{O}^{5}\right)$, on voit que la quantité d'acide phosphorique s'approche du minimum nécessaire.

La quantité de chaux est par contre de beaucoup inférieure à celle jugée indispensable.

Ce déficit, en aucun cas, ne pouvait ŝtre comblé par la chaux contenue dans l'eau servant à l'abreuvement des animaux (la « dureté » de l'eau utilisée était assez faible).

Dans ces conditions, nous pensons que e'est le déficit en calcium qui pourrait expliquer l'élimination urinaire du phosphore.

Pour couvrir les nécessités réclamées par le fonctionnement de la glande mammaire, l'organisme serait obligé de recourir à ses propres réserves de calcium.

Si cela est trai, il est fort probable qu'il fait appel en premier lieu à la chaux du squelette.

L'élimination du phosphore serait dans ce eas une conséquence nécessaire de la " fonte " des os.

(1) Le phosphore a été dosé par la méthode de Copaux. Le calcium a été précipité comme oxalate. Los deux dosages ont été faits sur les produits minéralisés à l'aide du mélange nitro-sulfurique. 